\title{
Neuroimaging of Rapid Eye Movement Sleep Behavior Disorder
}

\author{
Pierre-Alexandre Bourgouin ${ }^{*},{ }^{\dagger}$, Shady Rahayel ${ }^{*,+}$, Malo Gaubert ${ }^{*,+}$, \\ Dario Arnaldi ${ }^{\ddagger}$, Michele $\mathrm{Hu}^{\S}$, Anna Heidbreder" \\ David Rémillard-Pelchat ${ }^{*}{ }^{\dagger}$, Michele Terzaghi", \\ Jean-François Gagnon*,t,\#,1 \\ *Department of Psychology, Université du Québec à Montréal, Montreal, QC, Canada \\ †Center for Advanced Research in Sleep Medicine, CIUSSS-NÎM-Hôpital du Sacré-Cour de Montréal, \\ Montreal, QC, Canada \\ ${ }^{\ddagger}$ Clinical Neurology, DINOGMI, University of Genoa, IRCCS Ospedale Policlinico San Martino, \\ Genoa, Italy \\ ${ }^{\S}$ Department of Neurology, Nuffield Department of Clinical Neurosciences, Oxford University Hospitals \\ NHS Foundation Trust, Oxford, United Kingdom \\ IIInstitute for Sleep Medicine and Neuromuscular Disorders, University Hospital Muenster, \\ Muenster, Germany \\ "Sleep and Epilepsy Unit, IRCCS Mondino Foundation, Pavia, Italy \\ \#Research Center, Institut universitaire de gériatrie de Montréal, Montreal, QC, Canada \\ ${ }^{1}$ Corresponding author: e-mail address: gagnon.jean-françois.2@uqam.ca
}

\section{Contents}

1. Introduction 186

2. Molecular Radiotracer Imaging 187

$\begin{array}{ll}2.1 \text { Dopaminergic Imaging } & 187\end{array}$

2.2 Serotoninergic and Noradrenergic Imaging $\quad 190$

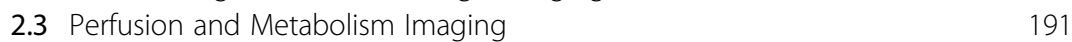

3. Functional Magnetic Resonance Imaging 194

4. Structural Magnetic Resonance Imaging 195

$\begin{array}{ll}4.1 \text { Gray Matter } & 195\end{array}$

4.2 White Matter 197

4.3 Other Techniques 198

5. Transcranial Sonography 199

6. Cardiac ${ }^{123}$ I-Metaiodobenzylguanidine Scintigraphy 200

7. Gut Imaging 201

8. Sleep Imaging 202

9. Methodological Considerations 202

10. Conclusion 203

References 205 


\section{Abstract}

Idiopathic rapid eye movement sleep behavior disorder (iRBD) is a parasomnia characterized by the loss of muscle atonia and the presence of undesirable motor manifestations during rapid eye movement sleep. Research findings have shown that RBD is a prodromal stage of synucleinopathies such as Parkinson's disease, dementia with Lewy bodies, and multiple system atrophy. A wide array of neuroimaging techniques have improved our understanding of the prodromal stage of these diseases in patients with iRBD, and identified potential biomarkers. In this chapter, we summarize current knowledge about functional and structural central and peripheral neuroimaging in iRBD, including cross-sectional and longitudinal studies using positron emission tomography, single-photon emission computed tomography, magnetic resonance imaging, and transcranial sonography. Current neuroimaging research has revealed several brain alterations in iRBD similar to those reported in synucleinopathies, thereby improving our understanding of the pathophysiology underlying the clinical presentation and progression of their prodromal stages. Moreover, some abnormalities detected by neuroimaging show promise as potential biomarkers to predict which individuals with iRBD may be at risk of conversion and therefore candidates for inclusion in future clinical trials of neuroprotection.

\section{INTRODUCTION}

Idiopathic rapid eye movement sleep behavior disorder (iRBD) is a parasomnia characterized by the loss of muscle atonia and the presence of abnormal movements during rapid eye movement sleep (American Academy of Sleep Medicine, 2014). Research has demonstrated clearly that $\mathrm{iRBD}$ is a prodromal stage of synucleinopathies including Parkinson's disease (PD), dementia with Lewy bodies, and multiple system atrophy. Indeed, long-term studies on large iRBD cohorts have estimated a 25-30\% risk of synucleinopathy at 3 years and 81-91\% at 14 years (Iranzo, FernándezArcos, et al., 2014; Postuma, Gagnon, Bertrand, Génier Marchand, \& Montplaisir, 2015; Schenck, Boeve, \& Mahowald, 2013). Moreover, iRBD patients present several clinical signs of synucleinopathies such as deficits in motor, cognitive, olfaction, color discrimination, and autonomic functions (Boeve, 2013; Högl, Stefani, \& Videnovic, 2018). However, the time course leading to conversion of $\mathrm{R}$ RD patients remains highly variable and the ability to predict whether iRBD patients will convert to PD, dementia with Lewy bodies or multiple system atrophy phenotypes is poor.

Neuroimaging techniques are a promising research avenue to identify new biomarkers that assess the risk of conversion toward synucleinopathies and clarify the clinical trajectory of $\mathrm{RRBD}$ patients. These objectives will be 
crucial in the coming years, during which we can expect the first human clinical trials for neuroprotective treatments at the earliest phase of disease. Moreover, neuroimaging allows for a better understanding of the prodromal stage of synucleinopathies, as it offers insight into in vivo functional and structural brain alterations in $\mathrm{R} B \mathrm{BD}$. This chapter aims to summarize the current state of knowledge of neuroimaging studies in iRBD.

\section{MOLECULAR RADIOTRACER IMAGING}

\subsection{Dopaminergic Imaging}

Nigrostriatal dopaminergic depletion is a hallmark of synucleinopathies and is likely responsible in part for the main motor symptoms of PD. Several cross-sectional studies have investigated the striatal dopaminergic function in iRBD patients using SPECT and PET imaging (Albin et al., 2000; Arnaldi, Famà, et al., 2015; Eisensehr et al., 2000, 2003; Frosini et al., 2017; Iranzo et al., 2010, 2011; Kim et al., 2010; Knudsen et al., 2018; Rupprecht et al., 2013; Stiasny-Kolster et al., 2005; Stokholm et al., 2017; Zoetmulder et al., 2016). Researchers investigated striatal postsynaptic dopamine D2-receptors using $\left[{ }^{123}\right.$ I]iodobenzamide (IBZM) SPECT, and found no differences between iRBD patients and healthy controls (Eisensehr et al., 2000, 2003). Others have studied striatal presynaptic dopaminergic function in iRBD with several tracers, including $\left[{ }^{11} \mathrm{C}\right]$ dihydrotetrabenazine (DTBZ), $\left[{ }^{18} \mathrm{~F}\right]$ dihydroxyphenylalanine (DOPA), $\left[{ }^{18} \mathrm{~F}\right]$ fluoro-L-m-tyrosine, $\left[{ }^{123} \mathrm{I}\right]$ Ioflupane (FP-CIT), and [ $\left.{ }^{123} \mathrm{I}\right] \mathrm{IPT}$. Studies in iRBD patients have found lower presynaptic striatal dopamine binding compared to controls but higher binding compared to PD patients using different tracers in PET and SPECT imaging (Albin et al., 2000; Arnaldi, Famà, et al., 2015; Eisensehr et al., 2000, 2003; Iranzo et al., 2010, 2011; Kim et al., 2010; Knudsen et al., 2018; Stokholm et al., 2017; Zoetmulder et al., 2016). Most studies have found a more pronounced reduction in dopamine binding in the putamen than in the caudate nucleus (Albin et al., 2000; Eisensehr et al., 2000; Iranzo et al., 2010, 2011; Knudsen et al., 2018; Stokholm et al., 2017), as typically seen in PD patients (Djang et al., 2012). However, one study compared nigrostriatal deafferentation using [ ${ }^{123}$ I] FP-CIT SPECT in 12 individuals with iRBD and de novo, drug-naïve PD patients with $(n=24)$ and without $(n=16)$ RBD (Arnaldi, De Carli, et al., 2015). Researchers found a more severe deafferentation in the caudate in $\mathrm{RRBD}$ and $\mathrm{PD}$ patients with RBD compared to $\mathrm{PD}$ patients without RBD, while the putaminal dopaminergic function progressively decreased from iRBD patients to PD patients without 
RBD and finally to PD patients with RBD (see Fig. 1 for an illustration). A recent meta-analysis concluded that tracer uptake in the putamen progressively decreased from controls to iRBD patients to PD patients without RBD, and to PD patients with RBD (Bauckneht et al., 2018). The same pattern was reported in the caudate but without differences between iRBD patients and PD patients without RBD (Bauckneht et al., 2018). On the other hand, a recent study found no difference in $\left[{ }^{123} \mathrm{I}\right] \mathrm{FP}-\mathrm{CIT}$ uptake at basal ganglia level between PD patients with probable RBD and PD patients without RBD symptoms, suggesting that a floor effect may be present (Pagano et al., 2018).

The percentage of iRBD patients with abnormal striatal dopamine transporter (DAT) binding varies greatly: from $12.5 \%$ to $45 \%$ in some studies (Iranzo et al., 2010, 2011; Kim et al., 2010; Knudsen et al., 2018; Rolinski et al., 2016; Rupprecht et al., 2013; Stiasny-Kolster et al., 2005), and from

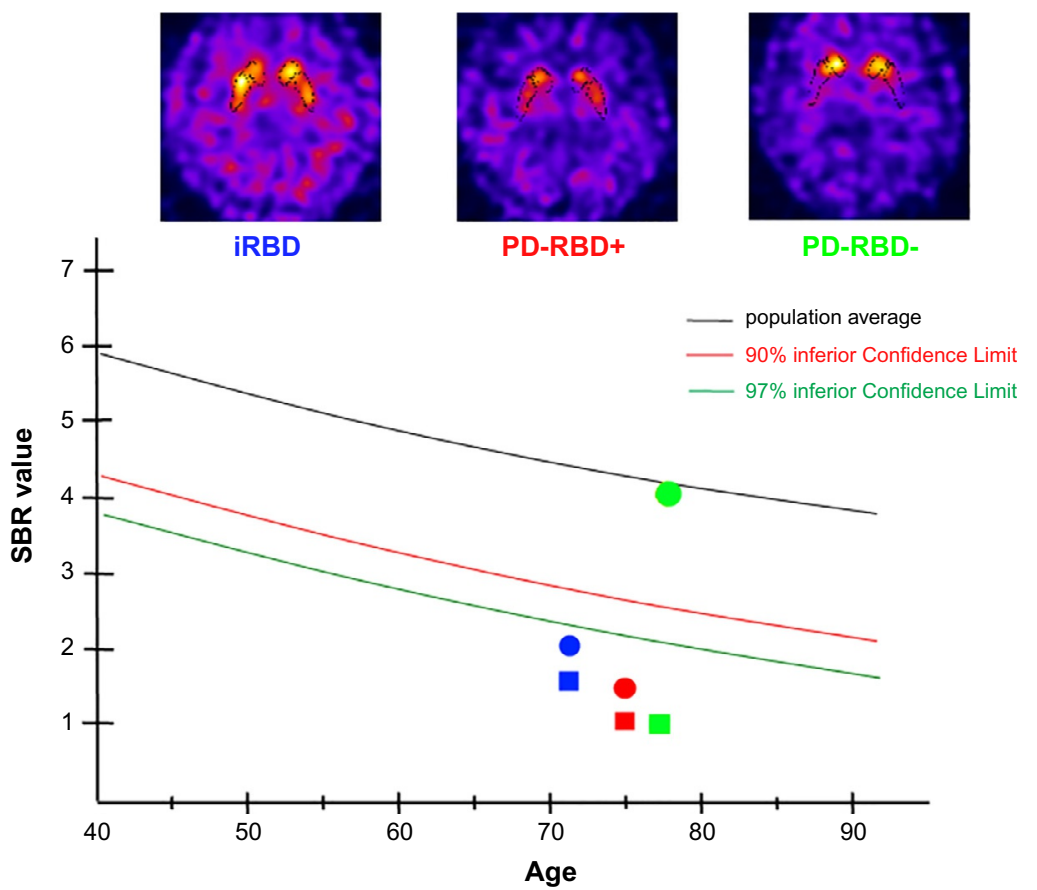

Fig. 1 Examples of $\left[{ }^{123}\right.$ I]FP-CIT-SPECT scans in idiopathic rapid eye movement sleep behavior disorder (iRBD; blue), Parkinson's disease (PD) with RBD (PD-RBD+; red) and PD without RBD (PD-RBD -; green). Comparison of specific to non-displaceable binding ratios (SBR) at putamen (square) and caudate (circle) levels with a dataset of normal subjects from the ENC-DAT database, analyzed with the Basal Ganglia Matching Tool (Nobili et al., 2013). 
59\% to 100\% in others (Eisensehr et al., 2000; Frosini et al., 2017; Iranzo, Santamaría, et al., 2017; Stokholm et al., 2017). Interestingly, striatal dopamine depletion becomes more severe as the severity of RBD nocturnal symptoms increases (more severe in clinically manifest RBD than in subclinical $\mathrm{RBD}$ ) and is also impaired in patients with longstanding iRBD without clear dementia and parkinsonism signs (Eisensehr et al., 2003; Iranzo, Stefani, et al., 2017). Recently, researchers also used [ $\left.{ }^{11} \mathrm{C}\right] \mathrm{PK} 11195$ PET to measure microglial activation, a marker of neuroinflammation (Stokholm et al., 2017). They found increased microglial activation in the left substantia nigra in iRBD patients compared to controls, which supports the hypothesis of a neuroinflammation component in the early neurodegenerative process of synucleinopathies.

Some studies have looked at the links between RBD motor signs and midbrain dopaminergic activity and found significant correlation between higher muscle activity during rapid eye movement sleep and reduction in striatal DAT binding (Eisensehr et al., 2003; Zoetmulder et al., 2016), although one study failed to identify such an association (Kim et al., 2010). Another study compared iRBD patients with and without abnormal striatal DAT binding and found higher Unified Parkinson's Disease Rating Scale (motor part) scores in patients with abnormal binding (Rupprecht et al., 2013). However, other studies were not able to replicate this finding (Bae et al., 2017; Iranzo et al., 2010, 2011). In one study using $\left[{ }^{18} \mathrm{~F}\right] \mathrm{DOPA}$ and $\left[{ }^{11} \mathrm{C}\right]$ raclopride PET in 11 patients with comorbid RBD and major depressive disorder and 10 controls, the authors found lower $\left[{ }^{18} \mathrm{~F}\right] \mathrm{DOPA}$ uptake in the putamen and caudate in patients (Wing et al., 2015). No between-group differences were found for the $\left[{ }^{11} \mathrm{C}\right]$ raclopride. In the same study, presynaptic dopamine dysfunction in the putamen was associated with severity of RBD symptoms on the questionnaire and higher muscle activity during rapid eye movement sleep.

To date, four studies have investigated presynaptic striatal dopamine binding using SPECT imaging in a longitudinal design (Iranzo et al., 2010, 2011; Iranzo, Santamaría, et al., 2017; Li, Kang, et al., 2017). The first study followed 43 iRBD patients for 2.5 years. At follow-up, 8 (19\%) of 43 patients had developed a synucleinopathy (Iranzo et al., 2010). In these patients, $\left[{ }^{123} \mathrm{I}\right]$ FP-CIT binding was up to 33\% lower at baseline in the entire striatum, 36\% in the putamen, and $40 \%$ in the caudate nucleus. Six of eight patients who converted had abnormal decreased striatal $\left[{ }^{123}\right.$ I]FP-CIT uptake at baseline. Another study by the same research team prospectively investigated the progression of striatal dopamine depletion in $20 \mathrm{iRBD}$ patients and 20 controls 
with serial DAT imaging over a 3-year period (Iranzo et al., 2011). They found greater striatal dopamine decline in $\mathrm{RBBD}$ patients in the bilateral putamen and left caudate nucleus. At the 3-year follow-up, three patients were diagnosed with PD. These patients had the lowest $\left[{ }^{123} \mathrm{I}\right] \mathrm{FP}-\mathrm{CIT}$ uptake at baseline. Two recent studies confirmed these results. The first followed 87 iRBD patients for a mean of 5.7 years (Iranzo, Santamaría, et al., 2017). During follow-up, 25 (29\%) patients had developed a synucleinopathy. Patients with abnormal DAT-SPECT at baseline were at higher risk of synucleinopathy than patients with normal DAT-SPECT $(20 \%$ versus $6 \%$ converted at 3 years, $33 \%$ versus $18 \%$ at 5 years). Moreover, a reduction of $\left[{ }^{123} \mathrm{I}\right] \mathrm{FP}-\mathrm{CIT}$ uptake at baseline in the putamen greater than $25 \%$ predicted conversion to a synucleinopathy within 3 years of follow-up. A similar study followed 34 iRBD patients who underwent DAT-SPECT ( $\left.{ }^{99 \mathrm{~m}} \mathrm{Tc}\right]$ TRODAT-1) at baseline over a period of 5 years (Li, Kang, et al., 2017). During follow-up, 18 (53\%) patients had developed a synucleinopathy. Patients with decreased $\left[{ }^{99 \mathrm{~m}} \mathrm{Tc}\right]$ TRODAT-1 binding in the left putamen had a higher risk of developing a synucleinopathy. These findings suggest that presynaptic striatal dopamine binding in the putamen is a potential biomarker of neurodegeneration in iRBD patients. However, available data in the literature on presynaptic radionuclide neuroimaging in iRBD patients are largely heterogeneous, particularly with respect to neuroimaging methodology (Bauckneht et al., 2018). Therefore, in the future, researchers will need to engage in large, multicentric studies to harmonize acquisition and reconstruction protocols as well as semiquantification procedures of presynaptic dopaminergic radionuclide imaging in $\mathrm{iRBD}$ so that these promising results can be translated into clinical practice.

\subsection{Serotoninergic and Noradrenergic Imaging}

Serotoninergic (raphe nuclei) and noradrenergic (locus coeruleus) systems are altered in synucleinopathies (Borghammer, 2018; Braak et al., 2003; Halliday, Holton, Revesz, \& Dickson, 2011; Jellinger, 2017). To date, few studies have investigated other monoaminergic neurotransmitters (serotoninergic and noradrenergic) in iRBD patients (Arnaldi, Famà, et al., 2015; Knudsen et al., 2018; Stokholm et al., 2018). In one study, researchers performed $\left[{ }^{123} \mathrm{I}\right] \mathrm{FP}-\mathrm{CIT}$ SPECT imaging of the serotonin transporter in the brainstem and thalamus in 20 iRBD patients and 23 controls (Arnaldi, Famà, et al., 2015). No between-group difference was 
found in ligand uptake at brainstem and thalamus level, suggesting that the serotonergic system is not impaired in iRBD patients. $\left[{ }^{123} \mathrm{I}\right] \mathrm{FP}-\mathrm{CIT}$ is a non-selective ligand, which mostly binds dopamine transporter at basal ganglia level and serotonin transporter at brainstem and thalamus level. Indeed, $\left[{ }^{123} \mathrm{I}\right] \mathrm{FP}-\mathrm{CIT}$ SPECT has been proposed as a reliable marker for the integrity of the brainstem serotonergic raphe nuclei, but only in a subgroup of PD patients (Qamhawi et al., 2015). In this study, raphe serotonin transporter availability was not associated with probable RBD in early PD. Furthermore, no difference in serotonin transporter binding at brainstem and striatal level, as evaluated by means of $\left[{ }^{11} \mathrm{C}\right] 3$-amino-4-(2-dimethylaminomethyl-phenylsulfaryl)-benzonitrile PET, was found between PD patients with probable RBD and PD patients without RBD symptoms (Kotagal et al., 2012), thus confirming that the serotonergic system is not associated with RBD.

In another study, researchers used $\left[{ }^{11} \mathrm{C}\right]$ methylreboxetine (MeNER) PET imaging to assess noradrenaline nerve terminals from the locus coeruleus to the thalamus and red nucleus in $17 \mathrm{iRBD}$ patients and 11 controls (Knudsen et al., 2018). They found trends toward lower $\left[{ }^{11} \mathrm{C}\right] \mathrm{MeNER}$ binding in patients in these two regions. In one recent study, researchers used $\left[{ }^{18} \mathrm{~F}\right] \mathrm{DOPA}$ and $\left[{ }^{11} \mathrm{C}\right] \mathrm{PK} 11195$ PET imaging to investigate extrastriatal monoaminergic function and microglial activation in $21 \mathrm{iRBD}$ patients and 29 controls (Stokholm et al., 2018). They found monoaminergic dysfunction in the thalamus in patients (mean reduction of $15 \%$ in $\left[{ }^{18}\right.$ F]DOPA $K_{\mathrm{i}}$ values), but without change in $\left[{ }^{11} \mathrm{C}\right] \mathrm{PK} 11195$ binding in this region. The authors suggest that the thalamic monoaminergic dysfunction in iRBD patients could be related to impaired locus coeruleus or dorsal raphe projections to the thalamus.

\subsection{Perfusion and Metabolism Imaging}

In cross-sectional studies, researchers used $\left[{ }^{99 \mathrm{~m}} \mathrm{Tc}\right] \mathrm{ECD}$ (ethylene cysteinate dimer) and $\left[{ }^{123}\right.$ I]IMP (iodoamphetamine) SPECT imaging to investigate regional cerebral blood flow in polysomnography-confirmed $\mathrm{R} B \mathrm{BD}$ patients compared to controls, and found several abnormalities (Hanyu et al., 2011; Mazza et al., 2006; Shirakawa et al., 2002; Vendette et al., 2011, 2012). One of these studies involved using $\left[{ }^{123}\right.$ I]IMP SPECT imaging in 20 iRBD patients and 7 controls (Shirakawa et al., 2002). This technique showed reduced regional cerebral blood flow in frontal lobes (upper portion) and pons in patients. In a second study, researchers used [ ${ }^{99 \mathrm{~m}} \mathrm{Tc}$ ]ECD SPECT 
imaging in eight iRBD patients and nine controls (Mazza et al., 2006). They reported decreased perfusion in patients' bilateral frontal and temporoparietal regions, and increased perfusion in the pons, bilateral putamen, and right hippocampus. The same research team confirmed these results in another sample, comprised of 20 iRBD patients and 20 controls (Vendette et al., 2011). Moreover, in patients, they found associations between reduced perfusion in the frontal and occipital regions and poorer performance on the color discrimination test, as well as between reduced perfusion in the anterior parahippocampal gyrus and loss of olfactory discrimination. They then compared subgroups of the last mentioned sample (Vendette et al., 2011), specifically $10 \mathrm{iRBD}$ patients with mild cognitive impairment, $10 \mathrm{iRBD}$ patients without mild cognitive impairment, and 20 controls (Vendette et al., 2012). Among these participants, they found reduced perfusion in occipital and temporal regions, and increased perfusion in the hippocampus, putamen, and paracentral gyrus in patients with mild cognitive impairment compared to patients without mild cognitive impairment and controls. Both subgroups of patients (with and without mild cognitive impairment) had reduced perfusion in the frontal lobes and increased perfusion in the hippocampus and parahippocampal regions compared to controls. These findings suggest that the brain perfusion abnormalities in $\mathrm{RBD}$ patients are more extensive in those with cognitive impairment. In another study, researchers performed $\left[{ }^{123} \mathrm{I}\right]$ IMP SPECT imaging in 24 iRBD patients and 18 controls (Hanyu et al., 2011), and found reduced regional cerebral blood flow in the parieto-occipital lobes (bilateral precuneus), right limbic lobe (uncus), and bilateral cerebellum of patients. They found no correlation between regional cerebral blood flow changes and polysomnographic variables or RBD duration.

Researchers also performed metabolic imaging with $\left[{ }^{18} \mathrm{~F}\right]$ fluorodeoxyglucose (FDG) PET in 21 iRBD patients and 21 controls (Ge et al., 2015). They found increased tracer uptake in the hippocampus and parahippocampal gyrus, cingulate, supplementary motor area, and pons, but reduced tracer uptake in the occipital cortex and lingual gyrus in patients. Chin electromyographic activity during rapid eye movement sleep correlated positively with metabolic activity in the hippocampus/parahippocampal gyrus, but negatively with activity in the posterior cingulate. RBD duration correlated positively with the metabolic activity in the anterior vermis, but negatively with activity in the medial frontal gyrus.

Other studies in PD have used spatial covariance analysis to identify abnormal metabolic brain networks that could accurately classify patients 
on an individual basis (Eidelberg, 2009; Huang et al., 2007; Ma, Tang, Spetsieris, Dhawan, \& Eidelberg, 2007; Tang, Poston, Dhawan, \& Eidelberg, 2010). Researchers used $\left[{ }^{18} \mathrm{~F}\right] \mathrm{FDG}$ PET to identify the PD-related covariance pattern, a highly reproducible abnormal metabolic pattern in PD (Ma et al., 2007). The topographical characteristics of this pattern were found to include increased metabolic activity in the pons, cerebellum, pallido-thalamic regions, and primary motor cortex, and reductions in the lateral premotor and parietal association regions (Eidelberg, 2009; Huang et al., 2007; Ma et al., 2007; Tang et al., 2010). In one study, researchers reported elevated PD-related covariance pattern expression in two iRBD cohorts, 1 of 10 patients (and 10 controls) who underwent $\left[{ }^{18} \mathrm{~F}\right] \mathrm{FDG}$ PET, and 1 of 17 patients (and 17 controls) who underwent $\left[{ }^{99 \mathrm{~m}} \mathrm{Tc}\right] \mathrm{ECD}$ SPECT (Holtbernd et al., 2014). Another research team confirmed these results and performed $\left[{ }^{18} \mathrm{~F}\right] \mathrm{FDG}$ PET in a cohort of $21 \mathrm{iRBD}$ patients and 21 controls to identify an iRBD-related covariance network (Wu et al., 2014). This network was characterized by increased metabolic activity in the pons, cerebellum, thalamus, hippocampus, medial frontal and sensorimotor areas, supramarginal, and inferior temporal gyrus, with reduced metabolic activity in the occipital and superior temporal regions. iRBD-related covariance network expression was high in early-stage PD patients with unilateral parkinsonism, but lower in more advanced PD patients (Wu et al., 2014). Another research team performed $\left[{ }^{18} \mathrm{~F}\right] \mathrm{FDG}$ PET in an independent cohort of $21 \mathrm{iRBD}$ patients and 19 controls, and identified a second iRBD-related covariance network (Meles et al., 2018). This latter iRBD-related covariance network was characterized by relative increased metabolism in the brainstem, cerebellum, thalamus, hippocampus, and sensorimotor cortex, with relative decreased metabolism in the occipito-parietal, temporal, and cingulate cortex. Therefore, the two iRBD-related covariance networks showed relatively similar patterns, and both largely overlapped the PD-related covariance pattern. However, the correlation between the two iRBD-related covariance networks and the PD-related covariance pattern scores on the one hand, and the clinical features on the other, showed some discrepancies. For instance, the first iRBD-related covariance network (Wu et al., 2014) was more associated with the severity of parkinsonism, the second iRBD-related covariance network (Meles et al., 2018) rather with the level of cognitive function. These findings suggest heterogeneous clinical presentation and progression in iRBD patients, which leads to a slightly different metabolic pattern, depending on the patient's clinical characteristics. 
To date, three longitudinal studies have investigated changes in perfusion and metabolism in iRBD patients (Dang-Vu et al., 2012; Holtbernd et al., 2014; Sakurai et al., 2014). The first study followed 20 iRBD patients who underwent $\left[{ }^{99 \mathrm{~m}} \mathrm{Tc}\right]$ ECD SPECT for a mean of 3 years (Dang-Vu et al., 2012). During follow-up, 10 patients had developed a synucleinopathy (PD in 5, dementia with Lewy bodies in 5). Researchers found increased perfusion in the hippocampus of patients who developed a synucleinopathy compared to patients who remained disease-free. In the former, hippocampal relative hyperperfusion was associated with other markers of neurodegeneration in $\mathrm{iRBD}$, such as motor symptoms and impaired color discrimination. In a second study, the authors conducted $\left[{ }^{123}\right.$ I]IMP SPECT at baseline and again after a 2-year follow-up in 9 iRBD patients and 18 controls (Sakurai et al., 2014). Despite a null rate of conversion at follow-up, they found decreased perfusion in the medial portions of the parieto-occipital and parieto-temporal regions. In a third study, the authors performed long-term follow-up (mean of 4.6 years) in $17 \mathrm{iRBD}$ patients who underwent $\left[{ }^{99 \mathrm{~m}} \mathrm{Tc}\right.$ ] ECD SPECT (Holtbernd et al., 2014). Eight patients developed a synucleinopathy ( $\mathrm{PD}$ in five, dementia with Lewy bodies in three). A model with age and elevated PD-related covariance pattern expression at time of imaging was a good predictor of phenoconversion in this cohort.

\section{FUNCTIONAL MAGNETIC RESONANCE IMAGING}

Resting-state functional magnetic resonance imaging dysfunction within the basal ganglia network is a feature of early PD, as distinct from other degenerative disorders, including Alzheimer's disease (Rolinski et al., 2015; Szewczyk-Krolikowski et al., 2014). In two studies, researchers performed resting-state functional magnetic resonance imaging in polysomnographyconfirmed iRBD patients (Ellmore et al., 2013; Rolinski et al., 2016). In the first study, researchers compared correlations of left and right substantia nigra time series using resting-state blood oxygen level-dependent functional magnetic resonance imaging between 10 controls, 10 iRBD, and 11 PD patients (Ellmore et al., 2013). They found correlations between the left substantia nigra and left putamen, which were higher for the control group and lower for the RBD group. Correlations were lower for the PD group versus $i R B D$ and control groups. In the second study, researchers studied 23 controls, $26 \mathrm{iRBD}$, and 48 early PD patients with resting-state functional magnetic resonance imaging; among these participants, 10 controls, $8 \mathrm{iRBD}$ and 10 PD patients also received [ ${ }^{123}$ I] ioflupane SPECT (Rolinski et al., 2016). 
They isolated resting-state networks from task-free functional magnetic resonance imaging data using independent component analysis, and showed widespread aberrant connectivity with the basal ganglia in iRBD patients before the onset of motor disease, which mirrors findings in the PD group. A perturbed basal ganglia connectivity network differentiated both iRBD and PD patients from controls with high sensitivity (96\%) and specificity (74-78\%) and with an area under the curve of 0.92 and 0.90 , respectively. The level of dopaminergic striatal loss in iRBD patients was intermediate between that in PD patients and controls.

\section{STRUCTURAL MAGNETIC RESONANCE IMAGING}

\subsection{Gray Matter}

Researchers have used different techniques to measure gray matter alterations in iRBD patients, including voxel-based morphometry, surface-based cortical thickness, subcortical volumetric, and vertex-based shape analysis.

In several studies, researchers have investigated gray matter volume using voxel-based morphometry in iRBD patients compared to controls, with inconsistent results. Some of these studies reported reduced gray matter volume in patients' frontal lobes, anterior cingulate gyri, cerebellum, pontine tegmentum, parahippocampal gyrus, and caudate nucleus (Hanyu et al., 2012; Rahayel et al., 2015; Rahayel, Postuma, Montplaisir, Bedetti, et al., 2018). In two others, researchers found no differences (Lee et al., 2014; Rolinski et al., 2016), whereas another research team reported increased gray matter density in the bilateral hippocampus in patients (Scherfler et al., 2011). In another study, researchers found that lower gray matter volume in the frontal lobes was associated with longer RBD duration and lower age of RBD symptoms onset (Rahayel, Postuma, Montplaisir, Bedetti, et al., 2018). Recently, researchers assessed cortical volume using a surface-based approach, which revealed cortical volume reductions in the left precentral and postcentral cortex only in iRBD patients with mild cognitive impairment (Rahayel, Postuma, Montplaisir, Génier Marchand, et al., 2018). In iRBD patients, lower performance in visuospatial abilities was associated with reduced cortical volume in the right lateral occipital cortex.

To date, three studies conducted by the same research team have investigated cortical thickness in iRBD patients compared to controls (Rahayel et al., 2015; Rahayel, Postuma, Montplaisir, Bedetti, et al., 2018; Rahayel, Postuma, Montplaisir, Génier Marchand, et al., 2018). In the first study, performed on 24 patients and 42 controls, these researchers found 
decreased cortical thickness in the frontal cortex, lingual gyrus, and fusiform gyrus (Rahayel et al., 2015). In a second study, performed in a larger sample of iRBD patients that included those studied previously (41 patients and 41 controls), they found thinning in the bilateral medial superior frontal, orbitofrontal and anterior cingulate cortices, and in the right dorsolateral primary motor cortexin patients (Rahayel, Postuma, Montplaisir, Bedetti, et al., 2018). In this study, they also found that reduced motor speed (finger tapping task) was associated with cortical thinning in the paracentral, postcentral, and superior parietal lobule cortices. In the third study, researchers divided patients into three groups according to their clinical status: $\mathrm{R} B \mathrm{BD}$ patients with mild cognitive impairment, iRBD patients without mild cognitive impairment, and controls (Rahayel, Postuma, Montplaisir, Génier Marchand, et al., 2018). Results revealed that patients with mild cognitive impairment had cortical thinning in the frontal, cingulate, temporal, and occipital cortices compared to the two other groups. Patients without mild cognitive impairment had cortical thinning restricted to the frontal cortex. Thinning in the left anterior temporal cortex (optimal cutoff of $2.66 \mathrm{~mm}$ ) best discriminated between patients with mild cognitive impairment and controls, with $76.5 \%$ sensitivity and $95.1 \%$ specificity (area under the curve of 0.91). In patients, lower performance in cognitive domains (i.e., attention and executive functions, learning and memory, and visuospatial abilities) was associated with cortical thinning in regions underlying these cognitive functions.

Four studies have investigated subcortical gray matter alterations more specifically (Ellmore et al., 2010; Lee et al., 2014; Rahayel, Postuma, Montplaisir, Bedetti, et al., 2018; Rahayel, Postuma, Montplaisir, Génier Marchand, et al., 2018). Using structure-based volumetric analysis of the striatum, researchers in one study found a lower volume of the bilateral putamen in five iRBD patients compared to seven controls (Ellmore et al., 2010). Another research team also performed volumetric analysis of several subcortical structures, in $15 \mathrm{iRBD}$ patients and 20 controls, and found no difference (Lee et al., 2014). More recently, researchers performed vertex-based shape and structure-based volumetric analysis in a larger group of $41 \mathrm{iRBD}$ patients and 41 controls (Rahayel, Postuma, Montplaisir, Bedetti, et al., 2018). In patients, they found extensive surface contraction in the external and internal segments of the left pallidum (with trends for surface contraction in both putamina) and a lower global volume in the right putamen. Shape contraction in the bilateral pallidum and right putamen was associated with 
reduced motor speed (finger tapping task). The last study examined shape abnormalities related to the presence of mild cognitive impairment in iRBD patients (Rahayel, Postuma, Montplaisir, Génier Marchand, et al., 2018). Researchers found contraction in patients with mild cognitive impairment, compared to patients without mild cognitive impairment (left putamen and thalamus) and controls (bilateral putamen and thalamus and left pallidum). Patients without mild cognitive impairment were similar to controls on subcortical shape measures. Lower performance in visuospatial abilities and verbal learning and memory in patients were associated with abnormal surface expansion in the right hippocampus.

Taken together, these results highlight the presence of cortical and subcortical gray matter abnormalities in $\mathrm{iRBD}$ patients, particularly in patients with cognitive impairment.

\subsection{White Matter}

To date, five studies have investigated white matter alterations with diffusion tensor imaging in $\mathrm{iRBD}$ patients compared to controls and reached inconsistent results (Mangia et al., 2017; Pyatigorskaya et al., 2017; Rahayel et al., 2015; Scherfler et al., 2011; Unger et al., 2010). In the first study, researchers looked at fractional anisotropy, axial diffusivity, and radial diffusivity using tract-based spatial statistics in 12 iRBD patients and 10 controls (Unger et al., 2010). They found several alterations in iRBD patients in the brainstem, right substantia nigra, left temporal lobe, fornix, olfactory region, internal capsule, corona radiata, and right visual stream. A second study looked at fractional anisotropy and mean diffusivity in $26 \mathrm{iRBD}$ patients and 14 controls (Scherfler et al., 2011). Researchers reported between-group differences restricted to the brainstem: increased mean diffusivity and reduced fractional anisotropy in the pontine tegmentum and midbrain in patients. In another study, researchers used tract-based spatial statistics in 24 iRBD patients and 41 controls (Rahayel et al., 2015). They found no significant differences in any diffusion tensor imaging metrics between patients and controls. Other researchers performed diffusion tensor imaging (fractional anisotropy and mean diffusivity) in subcortical regions in 8 iRBD patients and 10 controls and found no between-group differences after adjustment for age (Mangia et al., 2017). In the last study that investigated the substantia nigra in 19 iRBD patients and 18 controls, the authors found decreased fractional anisotropy in patients (Pyatigorskaya et al., 2017). 


\subsection{Other Techniques}

Other studies have used neuromelanin-sensitive, transverse relaxation rate $(\mathrm{R} 2 *)$, rotating frame relaxation methods, proton magnetic resonance spectroscopy, and susceptibility-weighted imaging to detect brain alterations in iRBD patients.

To date, three studies have been conducted using neuromelaninsensitive imaging in iRBD patients (Ehrminger et al., 2016; Knudsen et al., 2018; Pyatigorskaya et al., 2017). In one study, researchers investigated neuromelanin signal intensity in the locus coeruleus/subcoeruleus area in 21 iRBD patients and 21 controls (Ehrminger et al., 2016). Quantitative analysis showed reduced signal intensities in patients in the left and right coeruleus/subcoeruleus complexes. In the entire group of subjects (controls and patients), signal intensity was correlated with both percentage of rapid eye movement sleep without atonia and severity of RBD symptoms. Visual analyses of the signal classified patients with $82.5 \%$ sensitivity and $81 \%$ specificity. The same research team performed neuromelanin-sensitive imaging in the substantia nigra and found reduced volume and signal intensity in patients (Pyatigorskaya et al., 2017). The combination of both measures (neuromelanin volume and signal) correctly classified patients with $84 \%$ sensitivity and $89 \%$ specificity (area under the curve of 0.88 ). In another study, researchers confirmed the reduction in signal intensity previously reported in the locus coeruleus (locus coeruleus:pons ratios) in iRBD patients (Knudsen et al., 2018). Moreover, the reduction was similar to that found in PD patients.

In one study, researchers used transverse relaxation rate $(\mathrm{R} 2 *)$ to evaluate iron concentration in subcortical structures, namely the putamen, pallidum, caudate, thalamus, hippocampus, amygdala, brainstem, and substantia nigra (Lee et al., 2014). They found no significant differences between patients and controls (Lee et al., 2014). Similar results were obtained in the substantia nigra by Pyatigorskaya et al. (2017).

In a study described above, the authors also used rotating frame relaxation mapping methods, namely adiabatic $\mathrm{T}_{1} \rho$ and $\mathrm{T}_{2} \rho$ and Relaxation Along a Fictitious Field in the rotating frame of rank 4, to investigate subcortical regions (Mangia et al., 2017). After adjusting for age, they identified trends toward increased $T_{1} \rho$ length in the amygdala and toward increased $T_{2} \rho$ and Relaxation Along a Fictitious Field in the rotating frame of rank 4 lengths in the midbrain in patients, as compared to controls, suggesting neuronal loss in these regions. 
In two studies, researchers performed proton magnetic resonance spectroscopy to measure the concentration of metabolites, namely $\mathrm{N}$-acetylaspartate, creatine, choline-containing compound, and myoinositol, in localized brain areas, and obtained conflicting results (Iranzo et al., 2002; Zhang et al., 2016). In the first study, the authors found no significant differences in metabolic peaks and ratios in the pontine tegmentum and midbrain in 15 patients compared to 15 controls (Iranzo et al., 2002). In the second study, involving 18 iRBD patients and 29 controls, researchers investigated metabolite ratios in the pontine tegmentum (Zhang et al., 2016) and reported a reduction of Cho/Cr and NAA/Cho ratios in patients compared to controls.

To date, three research teams have reported on their studies using susceptibility-weighted imaging of the midbrain to visualize substantia nigra hyperintensity in controls, iRBD, and PD patients (Bae et al., 2017; De Marzi et al., 2016; Frosini et al., 2017). All found a loss of dorsolateral nigral hyperintensity in a significant proportion of patients $(60-80 \%$ in iRBD patients; $79-100 \%$ in PD patients) compared to controls (0-19\%). Moreover, dorsolateral nigral hyperintensity loss was associated with hyposmia (De Marzi et al., 2016) and reduced dopamine reuptake in the striatum (Bae et al., 2017; Frosini et al., 2017).

\section{TRANSCRANIAL SONOGRAPHY}

Researchers have conducted cross-sectional studies using transcranial sonography to estimate echogenicity of the substantia nigra in $\mathrm{RBD}$ patients compared to controls (Iranzo et al., 2010; Iwanami, Miyamoto, Miyamoto, Hirata, \& Takada, 2010; Li, Xue, et al., 2017; Shin, Joo, Kim, Dhong, \& Cho, 2013; Stockner et al., 2009; Vilas et al., 2015). Several authors reported substantia nigra hyperechogenicity in patients compared to controls (Iranzo et al., 2010; Iwanami et al., 2010; Shin et al., 2013; Stockner et al., 2009; Vilas et al., 2015), which was lower or of similar amplitude than in PD patients (Iwanami et al., 2010; Shin et al., 2013). The proportion of patients with pathological substantia nigra hyperechogenicity was higher in iRBD patients (32-63\%) than in controls (5-29\%) (Iranzo et al., 2010; Iwanami et al., 2010; Li, Xue, et al., 2017; Rupprecht et al., 2013; Shin et al., 2013; Stockner et al., 2009; Vilas et al., 2015).

One study assessed presynaptic dopaminergic function with $6-\left[{ }^{18} \mathrm{~F}\right]$ fluoro-meta-tyrosine PET in 19 iRBD patients, including 9 patients with abnormal substantia nigra hyperechogenicity (Miyamoto et al., 2012). These 
researchers found lower fluoro-meta-tyrosine uptake in the caudate and putamen in patients with abnormal substantia nigra hyperechogenicity compared to patients with normal values. However, in another study researchers reported no difference in $\left[{ }^{123} \mathrm{I}\right] \mathrm{FP}-\mathrm{CIT}$ SPECT uptake in striatal regions between patients with increased $(n=14)$ and normal $(n=25)$ substantia nigra echogenicity (Iranzo et al., 2010). Moreover, another study reported higher prevalence of motor impairment in iRBD patients with substantia nigra hyperechogenicity (Rupprecht et al., 2013), but most studies have failed to find associations between substantia nigra hyperechogenicity and other clinical symptoms.

Researchers also found hyperechogenicity in the basal ganglia in $50 \%$ (11 out of 22) of iRBD patients compared to 10\% (2 out of 21) of controls (Li, Xue, et al., 2017). In another study, researchers reported on their investigation of echogenicity in the brainstem raphe nuclei in relation with depression in 72 iRBD patients (Vilas et al., 2015). Thirty-two patients (44\%) were diagnosed with depression. Brainstem raphe hypoechogenicity was more frequent in patients with depression than those without (32\% versus $11 \%$ ).

One research team conducted two longitudinal studies to examine the predictive value of abnormal substantia nigra hyperechogenicity for synucleinopathy in iRBD patients (Iranzo et al., 2010; Iranzo, Stockner, et al., 2014). In the first study, 43 patients and 18 controls were followed for 2.5 years (Iranzo et al., 2010). At follow-up, eight patients had developed a synucleinopathy (PD in five, dementia with Lewy bodies in two, and multiple system atrophy in one) and five had substantia nigra hyperechogenicity at baseline. In a subsequent study, they followed 55 iRBD for 5 years (Iranzo, Stockner, et al., 2014). At follow-up, 21 patients had developed a neurodegenerative disease (PD in 11, dementia with Lewy bodies in 9, and multiple system atrophy in 1). No differences in measures of substantia nigra echogenicity were reported between the baseline and follow-up examination, nor between patients who converted compared to those who remained disease-free, suggesting that substantia nigra echogenicity is not a sensitive progression biomarker in $\mathrm{iRBD}$.

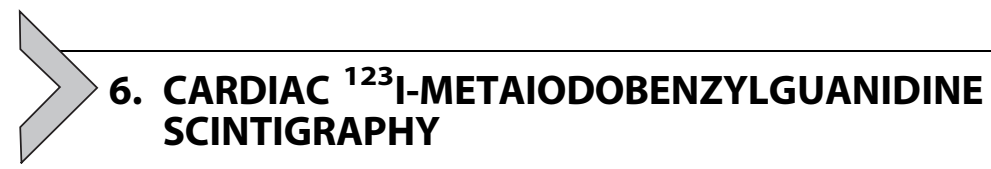

Four cross-sectional studies have been conducted to investigate cardiac $\left[{ }^{123} \mathrm{I}\right]$ metaiodobenzylguanidine $\left({ }^{123} \mathrm{I}-\mathrm{MIBG}\right)$ scintigraphy in $\mathrm{iRBD}$ patients compared to controls to assess cardiac sympathetic nerve endings 
(Kashihara, Imamura, \& Shinya, 2010; Knudsen et al., 2018; Miyamoto et al., 2006, 2008). These studies consistently revealed a reduced heart/ mediastinum $(\mathrm{H} / \mathrm{M})$ ratio or $\left[{ }^{123} \mathrm{I}\right]$ metaiodobenzylguanidine washout rate in patients compared to controls. The proportion of patients with abnormal values varied from $82 \%$ to 100\% (Knudsen et al., 2018; Miyamoto et al., 2006, 2008). Moreover, the results were similar to those of PD and dementia with Lewy bodies patients (Kashihara et al., 2010; Knudsen et al., 2018; Miyamoto et al., 2006, 2008). A single longitudinal study has been conducted using $\left[{ }^{123} \mathrm{I}\right]$ metaiodobenzylguanidine scintigraphy in iRBD patients. In another study, researchers followed 15 iRBD patients for a mean of 2.5 years, and found no significant differences between $\left[{ }^{123} \mathrm{I}\right]$ metaiodobenzylguanidine scintigraphy measures at baseline and follow-up (Miyamoto, Miyamoto, Iwanami, \& Hirata, 2011). Taken together, these results suggest that cardiac sympathetic denervation is abnormal early in the course of the disease, but is likely not a sensitive progression biomarker in $\mathrm{RBD}$.

\section{GUT IMAGING}

Researchers hypothesize that alpha-synuclein aggregates form in nerve terminals of the gastrointestinal lining many years before the apparition of PD motor symptoms (Borghammer, 2018; Braak et al., 2003; Stokholm, Danielsen, Hamilton-Dutoit, \& Borghammer, 2016). In one study, researchers used $\left[{ }^{11} \mathrm{C}\right]$ donepezil PET and computed tomography to assess cholinergic parasympathetic innervation of the gut in $20 \mathrm{iRBD}$ patients and 16 controls (Knudsen et al., 2018). They reported decreased $\left[{ }^{11} \mathrm{C}\right]$ donepezil uptake in the small intestine and colon in patients compared to controls. They observed a similar reduction in $18 \mathrm{PD}$ patients. Interestingly, as mentioned previously in this chapter, these researchers also performed multimodal imaging of the cardiac sympathetic innervation, locus coeruleus (pigmented neurons and thalamic noradrenergic nerve terminals), and nigrostriatal (dopamine storage capacity) functions. They calculated pathology indices across the five imaging modalities according to controls (set at $0 \%$ ) and PD (set at 100\%) average values. Globally, among iRBD patients, they found severe neural alterations (between 100\% and 150\%) in the peripheral autonomic system (gut and cardiac innervation) and locus coeruleus (neuromelanin-sensitive), followed by noradrenergic thalamic denervation (73\%), and putaminal dopaminergic storage capacity (21\%). Their findings support the caudorostral staging system of neuropathology in PD and dementia with Lewy bodies (Adler \& Beach, 2016; Braak et al., 2003). 


\section{SLEEP IMAGING}

The polysomnographic characteristics of RBD are the loss of normal rapid eye movement sleep muscle atonia and the presence of abnormal and undesirable motor activity during this sleep stage. In two studies, researchers performed ictal $\left[{ }^{99 \mathrm{~m}} \mathrm{Tc}\right] \mathrm{ECD}$ SPECT during episodes of RBD in a total of five RBD cases (iRBD in one, PD associated with RBD in one, narcolepsy associated with RBD in two, and multiple system atrophy associated with RBD in one) (Dauvilliers et al., 2011; Mayer, Bitterlich, Kuwert, Ritt, \& Stefan, 2015). A common finding showed activation of the cortical premotor supplementary area during dream-enactment episodes compared to wakefulness. Interestingly, structures in the basal ganglia were not activated during RBD episodes in either study. This result confirms the hypothesis that movements during RBD episodes are generated by the motor cortex and follow the pyramidal tract, bypassing the extrapyramidal system (De Cock et al., 2007). Indeed, in PD patients, researchers have observed that $\mathrm{RBD}$ episodes during rapid eye movement sleep are characterized by jerky but fast and coordinated movements, which contrast with the bradykinesia due to parkinsonism typically observed during the waking state.

\section{METHODOLOGICAL CONSIDERATIONS}

It is important to highlight several methodological concerns regarding the studies described above. First, most were performed on relatively small cohorts of $\mathrm{iRBD}$ patients, which may limit the statistical power to detect alterations or relationships with other markers of neurodegeneration in this population. Future studies should therefore consider a multicentric approach to generate larger samples (Bauckneht et al., 2018). However, such studies present technical challenges, due to differences in neuroimaging modality acquisition and clinical assessment of patients. Discrepancies between neuroimaging studies also stem from both statistical and methodological considerations. Indeed, the parameters used during neuroimaging processing are highly inconsistent between studies, which may limit reproducibility of results. Second, RBD has a heterogeneous clinical presentation and progression, which could also account for some of the discrepancies reported in the literature. This clinical heterogeneity is also evident in most of the studies described above that use PD as a comparison group. Indeed, these studies compared $\mathrm{R} B \mathrm{BD}$ patients to $\mathrm{PD}$ patients as a whole group 
(for example, without separating PD patients on the basis of the presence or absence of mild cognitive impairment or RBD). Yet, researchers have now documented that PD patients with RBD have a more severe clinical presentation and progression (including cognitive decline, autonomic dysfunction, motor symptoms, and extensive brain alterations) (Fereshtehnejad \& Postuma, 2017; Jozwiak et al., 2017; Pagano et al., 2018; Zhu, Xie, Hu, \& Wang, 2017). The lack of comparison to this particular subtype of PD is important, as PD patients with concomitant RBD will be the clinical phenotype exhibited by RBD patients converting to PD. Third, in several of the studies, researchers used a single neuroimaging technique to investigate peripheral and central brain alterations in iRBD patients; future multimodal approaches, combining several techniques in a single study, may reveal how structural and functional abnormalities associate with each other. In addition, several of the studies discussed above were designed as cross-sectional; despite being of interest for understanding the neuropathological mechanisms of iRBD and identifying potential biomarkers, the intrinsic nature of RBD, as a neurodegenerative disease, demands that researchers follow patients longitudinally in order to better assess the predictive value of the different neuroimaging modalities (Heller et al., 2017). However, we recognize the importance of cross-sectional findings, especially in future meta-analyses. With this in mind, it is also important to recall that several of the neuroimaging studies published to date were conducted on the same iRBD cohorts; consequently, replication in independent cohorts is crucial to ensure that abnormal findings can indeed be attributed to RBD pathology rather than to peculiarities of the samples.

\section{CONCLUSION}

Neuroimaging modalities reveal numerous structural and functional brain alterations in $\mathrm{RBD}$ patients that mirror and precede those found in synucleinopathies (Table 1). These findings confirm iRBD as a prodromal stage and the presence of ongoing peripheral and central neuronal dysfunction in these patients. Moreover, iRBD patients who present cognitive impairment appear to be at a more advanced stage of neurodegeneration, as revealed by reports of more extensive cortical and subcortical neuroimaging alterations in these patients. To date, dopaminergic function in the striatum as measured using SPECT seems to be the most promising biomarker for predicting the development of PD in iRBD. However, future 
Table 1 Main Finding for Each Neuroimaging Technique

Technique Main Finding

Molecular radiotracer imaging

Dopaminergic Presynaptic striatal dopamine binding in the putamen is a potential biomarker of neurodegeneration

Serotoninergic and Inconsistent results depending on the radiotracers used noradrenergic

\begin{tabular}{ll}
\hline $\begin{array}{c}\text { Perfusion and } \\
\text { metabolism }\end{array}$ & $\begin{array}{l}\text { Pattern of cortical and subcortical increased and } \\
\text { decreased perfusion/metabolism similar to that } \\
\text { reported in Parkinson's disease }\end{array}$ \\
\hline
\end{tabular}

Functional magnetic Perturbed basal ganglia connectivity network resonance imaging

Structural magnetic resonance imaging

Gray matter

Reduced thinning, volume, and shape in cortical and subcortical regions related to motor and cognitive functions

\begin{tabular}{ll}
\hline \multicolumn{1}{c}{ White matter } & Inconsistent results \\
\hline $\begin{array}{c}\text { Other techniques } \\
\text { (neuromelanin) }\end{array}$ & $\begin{array}{l}\text { Reduced signal in the substantia nigra and locus } \\
\text { coeruleus }\end{array}$ \\
\hline Transcranial sonography & Hyperechogenicity in the substantia nigra \\
\hline $\begin{array}{l}\text { Cardiac }\left[{ }^{123} \mathrm{I}\right]- \\
\text { metaiodobenzylguanidine } \\
\text { scintigraphy }\end{array}$ & $\begin{array}{l}\text { Cardiac sympathetic denervation early in the course of } \\
\text { the disease }\end{array}$ \\
\hline Sleep imaging & $\begin{array}{l}\text { Severe decreased }\left[{ }^{11} \mathrm{C}\right]-\text { donepezil uptake in the small } \\
\text { intestine and colon }\end{array}$ \\
\hline
\end{tabular}

studies will have to determine whether this modality allows us to predict which synucleinopathy an iRBD subject is most likely to develop (i.e., PD versus dementia with Lewy bodies versus multiple system atrophy). Further neuroimaging studies are therefore needed so that researchers can develop and validate biomarkers that would facilitate more accurate identification of the phenotypic entities that $\mathrm{R} B \mathrm{BD}$ subjects are likely to develop. Several neuroimaging modalities (perfusion imaging, metabolic imaging, functional and structural magnetic resonance imaging) have biomarker 
potential but still need validation from prospective and longitudinal studies. In the future, combining complementary imaging approaches with clinical measures in a multimodal approach would be an interesting avenue of research to identify patients at high risk of synucleinopathy.

\section{REFERENCES}

Adler, C. H., \& Beach, T. G. (2016). Neuropathological basis of nonmotor manifestations of Parkinson's disease. Movement Disorders, 31, 1114-1119.

Albin, R. L., Koeppe, R. A., Chervin, R. D., Consens, F. B., Wernette, K., Frey, K. A., et al. (2000). Decreased striatal dopaminergic innervation in REM sleep behavior disorder. Neurology, 55, 1410-1412.

American Academy of Sleep Medicine. (2014). International classification of sleep disordersThird edition (ICSD-3). AASM Resource Library.

Arnaldi, D., De Carli, F., Picco, A., Ferrara, M., Accardo, J., Bossert, I., et al. (2015). Nigro-caudate dopaminergic deafferentation: A marker of REM sleep behavior disorder? Neurobiology of Aging, 36, 3300-3305.

Arnaldi, D., Famà, F., De Carli, F., Morbelli, S., Ferrara, M., Picco, A., et al. (2015). The role of the serotonergic system in REM sleep behavior disorder. Sleep, 38, 1505-1509.

Bae, Y. J., Kim, J.-M., Kim, K. J., Kim, E., Park, H. S., Kang, S. Y., et al. (2017). Loss of substantia nigra hyperintensity at 3.0-T MR imaging in idiopathic REM sleep behavior disorder: Comparison with 123I-FP-CIT SPECT. Radiology, 287, 285-293.

Bauckneht, M., Chincarini, A., De Carli, F., Terzaghi, M., Morbelli, S., Nobili, F., et al. (2018). Presynaptic dopaminergic neuroimaging in REM sleep behavior disorder: A systematic review and meta-analysis. Sleep Medicine Reviews, 41, 266-274.

Boeve, B. F. (2013). Idiopathic REM sleep behaviour disorder in the development of Parkinson's disease. Lancet Neurology, 12, 469-482.

Borghammer, P. (2018). How does parkinson's disease begin? Perspectives on neuroanatomical pathways, prions, and histology. Movement Disorders, 33, 48-57.

Braak, H., Del Tredici, K., Rub, U., de Vos, R. A., Jansen Steur, E. N., \& Braak, E. (2003). Staging of brain pathology related to sporadic Parkinson's disease. Neurobiology of Aging, 24, 197-211.

Dang-Vu, T. T., Gagnon, J. F., Vendette, M., Soucy, J. P., Postuma, R. B., \& Montplaisir, J. (2012). Hippocampal perfusion predicts impending neurodegeneration in REM sleep behavior disorder. Neurology, 79, 2302-2306.

Dauvilliers, Y., Boudousq, V., Lopez, R., Gabelle, A., De Cock, V. C., Bayard, S., et al. (2011). Increased perfusion in supplementary motor area during a REM sleep behaviour episode. Sleep Medicine, 12, 531-532.

De Cock, V. C., Vidailhet, M., Leu, S., Texeira, A., Apartis, E., Elbaz, A., et al. (2007). Restoration of normal motor control in Parkinson's disease during REM sleep. Brain, 130, 450-456.

De Marzi, R., Seppi, K., Högl, B., Müller, C., Scherfler, C., Stefani, A., et al. (2016). Loss of dorsolateral nigral hyperintensity on 3.0 tesla susceptibility-weighted imaging in idiopathic rapid eye movement sleep behavior disorder. Annals of Neurology, 79, 1026-1030.

Djang, D. S., Janssen, M. J., Bohnen, N., Booij, J., Henderson, T. A., Herholz, K., et al. (2012). SNM practice guideline for dopamine transporter imaging with 123I-ioflupane SPECT 1.0. Journal of Nuclear Medicine, 53, 154-163.

Ehrminger, M., Latimier, A., Pyatigorskaya, N., Garcia-Lorenzo, D., Leu-Semenescu, S., Vidailhet, M., et al. (2016). The coeruleus/subcoeruleus complex in idiopathic rapid eye movement sleep behaviour disorder. Brain, 139, 1180-1188. 
Eidelberg, D. (2009). Metabolic brain networks in neurodegenerative disorders: A functional imaging approach. Trends in Neurosciences, 32, 548-557.

Eisensehr, I., Linke, R., Noachtar, S., Schwarz, J., Gildehaus, F. J., \& Tatsch, K. (2000). Reduced striatal dopamine transporters in idiopathic rapid eye movement sleep behaviour disorder-Comparison with Parkinson's disease and controls. Brain, 123, 1155-1160.

Eisensehr, I., Linke, R., Tatsch, K., Kharraz, B., Gildehaus, J. F., Wetter, C. T., et al. (2003). Increased muscle activity during rapid eye movement sleep correlates with decrease of striatal presynaptic dopamine transporters. IPT and IBZM SPECT imaging in subclinical and clinically manifest idiopathic REM sleep behavior disorder, Parkinson's disease, and controls. Sleep, 26, 507-512.

Ellmore, T. M., Castriotta, R. J., Hendley, K. L., Aalbers, B. M., Furr-Stimming, E., Hood, A. J., et al. (2013). Altered nigrostriatal and nigrocortical functional connectivity in rapid eye movement sleep behavior disorder. Sleep, 36, 1885-1892.

Ellmore, T. M., Hood, A. J., Castriotta, R. J., Stimming, E. F., Bick, R. J., \& Schiess, M. C. (2010). Reduced volume of the putamen in REM sleep behavior disorder patients. Parkinsonism and Related Disorders, 16, 645-649.

Fereshtehnejad, S. M., \& Postuma, R. B. (2017). Subtypes of Parkinson's disease: What do they tell us about disease progression? Current Neurology and Neuroscience Reports, 17, 34. http://doi:10.1007/s11910-017-0738-x.

Frosini, D., Cosottini, M., Donatelli, G., Costagli, M., Biagi, L., Pacchetti, C., et al. (2017). Seven tesla MRI of the substantia nigra in patients with rapid eye movement sleep behavior disorder. Parkinsonism and Related Disorders, 43, 105-109.

Ge, J., Wu, P., Peng, S., Yu, H., Zhang, H., Guan, Y., et al. (2015). Assessing cerebral glucose metabolism in patients with idiopathic rapid eye movement sleep behavior disorder. Journal of Cerebral Blood Flow and Metabolism, 35, 2062-2069.

Halliday, G. M., Holton, J. L., Revesz, T., \& Dickson, D. W. (2011). Neuropathology underlying clinical variability in patients with synucleinopathies. Acta Neuropathologica, 122, 187-204.

Hanyu, H., Inoue, Y., Sakurai, H., Kanetaka, H., Nakamura, M., Miyamoto, T., et al. (2011). Regional cerebral blood flow changes in patients with idiopathic REM sleep behavior disorder. European Journal of Neurology, 18, 784-788.

Hanyu, H., Inoue, Y., Sakurai, H., Kanetaka, H., Nakamura, M., Miyamoto, T., et al. (2012). Voxel-based magnetic resonance imaging study of structural brain changes in patients with idiopathic REM sleep behavior disorder. Parkinsonism and Related Disorders, $18,136-139$.

Heller, J., Brcina, N., Dogan, I., Holtbernd, F., Romanzetti, S., Schulz, J. B., et al. (2017). Brain imaging findings in idiopathic REM sleep behavior disorder (RBD)-A systematic review on potential biomarkers for neurodegeneration. Sleep Medicine Reviews, 34, $23-33$.

Högl, B., Stefani, A., \& Videnovic, A. (2018). Idiopathic REM sleep behaviour disorder and neurodegeneration-An update. Nature Reviews Neurology, 14, 40-55.

Holtbernd, F., Gagnon, J.-F., Postuma, R. B., Ma, Y., Tang, C. C., Feigin, A., et al. (2014). Abnormal metabolic network activity in REM sleep behavior disorder. Neurology, 82, 620-627.

Huang, C., Tang, C., Feigin, A., Lesser, M., Ma, Y., Pourfar, M., et al. (2007). Changes in network activity with the progression of Parkinson's disease. Brain, 130, 1834-1846.

Iranzo, A., Fernández-Arcos, A., Tolosa, E., Serradell, M., Molinuevo, J. L., Valldeoriola, F., et al. (2014). Neurodegenerative disorder risk in idiopathic REM sleep behavior disorder: Study in 174 patients. PLoS One, 9, e89741. 
Iranzo, A., Lomeña, F., Stockner, H., Valldeoriola, F., Vilaseca, I., Salamero, M., et al. (2010). Decreased striatal dopamine transporter uptake and substantia nigra hyperechogenicity as risk markers of synucleinopathy in patients with idiopathic rapid-eyemovement sleep behaviour disorder: A prospective study. The Lancet Neurology, 9, 1070-1077.

Iranzo, A., Santamaria, J., Pujol, J., Moreno, A., Deus, J., \& Tolosa, E. (2002). Brainstem proton magnetic resonance spectroscopy in idopathic REM sleep behavior disorder. Sleep, 25, 867-870.

Iranzo, A., Santamaría, J., Valldeoriola, F., Serradell, M., Salamero, M., Gaig, C., et al. (2017). Dopamine transporter imaging deficit predicts early transition to synucleinopathy in idiopathic rapid eye movement sleep behavior disorder. Annals of Neurology, 82, 419-428.

Iranzo, A., Stefani, A., Serradell, M., Marti, M. J., Lomena, F., Mahlknecht, P., et al. (2017). Characterization of patients with longstanding idiopathic REM sleep behavior disorder. Neurology, 89, 242-248.

Iranzo, A., Stockner, H., Serradell, M., Seppi, K., Valldeoriola, F., Frauscher, B., et al. (2014). Five-year follow-up of substantia nigra echogenicity in idiopathic REM sleep behavior disorder. Movement Disorders, 29, 1774-1780.

Iranzo, A., Valldeoriola, F., Lomeña, F., Molinuevo, J. L., Serradell, M., Salamero, M., et al. (2011). Serial dopamine transporter imaging of nigrostriatal function in patients with idiopathic rapid-eye-movement sleep behaviour disorder: A prospective study. The Lancet Neurology, 10, 797-805.

Iwanami, M., Miyamoto, T., Miyamoto, M., Hirata, K., \& Takada, E. (2010). Relevance of substantia nigra hyperechogenicity and reduced odor identification in idiopathic REM sleep behavior disorder. Sleep Medicine, 11, 361-365.

Jellinger, K. A. (2017). Neuropathology of nonmotor symptoms of Parkinson's disease. International Review of Neurobiology, 133, 13-62.

Jozwiak, N., Postuma, R. B., Montplaisir, J., Latreille, V., Panisset, M., Chouinard, S., et al. (2017). REM sleep behavior disorder and cognitive impairment in Parkinson's disease. Sleep, 40(8), zsx101. http://doi:10.1093/sleep/zsx101.

Kashihara, K., Imamura, T., \& Shinya, T. (2010). Cardiac 123I-MIBG uptake is reduced more markedly in patients with REM sleep behavior disorder than in those with early stage Parkinson's disease. Parkinsonism and Related Disorders, 16, 252-255.

Kim, Y. K., Yoon, I. Y., Kim, J. M., Jeong, S. H., Kim, K. W., Shin, Y. K., et al. (2010). The implication of nigrostriatal dopaminergic degeneration in the pathogenesis of REM sleep behavior disorder. European Journal of Neurology, 17, 487-492.

Knudsen, K., Fedorova, T. D., Hansen, A. K., Sommerauer, M., Otto, M., Svendsen, K. B., et al. (2018). In-vivo staging of pathology in REM sleep behaviour disorder: A multimodality imaging case-control study. The Lancet Neurology, 17, 618-628.

Kotagal, V., Albin, R. L., Müller, M. L., Koeppe, R. A., Chervin, R. D., Frey, K. A., et al. (2012). Symptoms of rapid eye movement sleep behavior disorder are associated with cholinergic denervation in Parkinson disease. Annals of Neurology, 71, 560-568.

Lee, J. H., Han, Y. H., Cho, J. W., Lee, J. S., Lee, S. J., Kim, D. J., et al. (2014). Evaluation of brain iron content in idiopathic REM sleep behavior disorder using quantitative magnetic resonance imaging. Parkinsonism and Related Disorders, 20, 776-778.

Li, Y., Kang, W., Yang, Q., Zhang, L., Zhang, L., Dong, F., et al. (2017). Predictive markers for early conversion of iRBD to neurodegenerative synucleinopathy diseases. Neurolog $\gamma$, $88,1493-1500$.

Li, X., Xue, S., Jia, S., Zhou, Z., Qiao, Y., Hou, C., et al. (2017). Transcranial sonography in idiopathic REM sleep behavior disorder and multiple system atrophy. Psychiatry and Clinical Neurosciences, 71, 238-246. 
Ma, Y., Tang, C., Spetsieris, P. G., Dhawan, V., \& Eidelberg, D. (2007). Abnormal metabolic network activity in Parkinson's disease: Test-retest reproducibility. Journal of Cerebral Blood Flow and Metabolism, 27, 597-605.

Mangia, S., Svatkova, A., Mascali, D., Nissi, M. J., Burton, P. C., Bednarik, P., et al. (2017). Multi-modal brain MRI in subjects with PD and iRBD. Frontiers in Neuroscience, 11, 709. http://doi:10.3389/fnins.2017.00709. eCollection 2017.

Mayer, G., Bitterlich, M., Kuwert, T., Ritt, P., \& Stefan, H. (2015). Ictal SPECT in patients with rapid eye movement sleep behaviour disorder. Brain, 38, 1263-1270.

Mazza, S., Soucy, J. P., Gravel, P., Michaud, M., Postuma, R., Massicotte-Marquez, J., et al. (2006). Assessing whole brain perfusion changes in patients with REM sleep behavior disorder. Neurology, 67, 1618-1622.

Meles, S. K., Renken, R. J., Janzen, A. H. O., Vadasz, D., Pagani, M., Arnaldi, D., et al. (2018). The metabolic pattern of idiopathic REM sleep behavior disorder reflects early-stage Parkinson disease. Journal of Nuclear Medicine, 59, 1437-1444.

Miyamoto, T., Miyamoto, M., Inoue, Y., Usui, Y., Suzuki, K., \& Hirata, K. (2006). Reduced cardiac 123I-MIBG scintigraphy in idiopathic REM sleep behavior disorder. Neurology, 67, 2236-2238.

Miyamoto, T., Miyamoto, M., Iwanami, M., \& Hirata, K. (2011). Follow-up study of cardiac ${ }^{123} \mathrm{I}-\mathrm{MIBG}$ scintigraphy in idiopathic REM sleep behavior disorder. European Journal of Neurology, 18, 1275-1278.

Miyamoto, M., Miyamoto, T., Iwanami, M., Muramatsu, S., Asari, S., Nakano, I., et al. (2012). Preclinical substantia nigra dysfunction in rapid eye movement sleep behaviour disorder. Sleep Medicine, 13, 102-106.

Miyamoto, T., Miyamoto, M., Suzuki, K., Nishibayashi, M., Iwanami, M., \& Hirata, K. (2008). 123I-MIBG cardiac scintigraphy provides clues to the underlying neurodegenerative disorder in idiopathic REM sleep behavior disorder. Sleep, 31, 717-723.

Nobili, F., Naseri, M., De Carli, F., Asenbaum, S., Booij, J., Darcourt, J., et al. (2013). Automatic semi-quantification of $\left[{ }^{123}\right.$ I] FP-CIT SPECT scans in healthy volunteers using BasGan version 2: Results from the ENC-DAT database. European Journal of Nuclear Medicine and Molecular Imaging, 40, 565-573.

Pagano, G., De Micco, R., Yousaf, T., Wilson, H., Chandra, A., \& Politis, M. (2018). REM behavior disorder predicts motor progression and cognitive decline in Parkinson disease. Neurology, 91, e894-e905. http://doi:10.1212/WNL.0000000000006134.

Postuma, R. B., Gagnon, J. F., Bertrand, J. A., Génier Marchand, D., \& Montplaisir, J. Y. (2015). Parkinson risk in idiopathic REM sleep behavior disorder: Preparing for neuroprotective trials. Neurology, 84, 1104-1113.

Pyatigorskaya, N., Gaurav, R., Arnaldi, D., Leu-Semenescu, S., Yahia-Cherif, L., Valabregue, R., et al. (2017). Magnetic resonance imaging biomarkers to assess substantia nigra damage in idiopathic rapid eye movement sleep behavior disorder. Sleep, 40(11), zsx149. http://doi:10.1093/sleep/zsx149.

Qamhawi, Z., Towey, D., Shah, B., Pagano, G., Seibyl, J., Marek, K., et al. (2015). Clinical correlates of raphe serotonergic dysfunction in early Parkinson's disease. Brain, 138, 2964-2973.

Rahayel, S., Montplaisir, J., Monchi, O., Bedetti, C., Postuma, R. B., Brambati, S., et al. (2015). Patterns of cortical thinning in idiopathic rapid eye movement sleep behavior disorder. Movement Disorders, 30, 680-687.

Rahayel, S., Postuma, R. B., Montplaisir, J., Bedetti, C., Brambati, S., Carrier, J., et al. (2018). Abnormal gray matter shape, thickness, and volume in the motor corticosubcortical loop in idiopathic rapid eye movement sleep behavior disorder: Association with clinical and motor features. Cerebral Cortex, 28, 658-671.

Rahayel, S., Postuma, R. B., Montplaisir, J., Génier Marchand, D., Escudier, F., Gaubert, M., et al. (2018). Cortical and subcortical gray matter bases of cognitive deficits in REM sleep behavior disorder. Neurology, 90, e1759-e1770. 
Rolinski, M., Griffanti, L., Piccini, P., Roussakis, A. A., Szewczyk-Krolikowski, K., Menke, R. A., et al. (2016). Basal ganglia dysfunction in idiopathic REM sleep behaviour disorder parallels that in early Parkinson's disease. Brain, 139, 2224-2234.

Rolinski, M., Griffanti, L., Szewczyk-Krolikowski, K., Menke, R. A. L., Wilcock, G. K., Filippini, N., et al. (2015). Aberrant functional connectivity within the basal ganglia of patients with Parkinson's disease. NeuroImage. Clinical, 8, 126-132.

Rupprecht, S., Walther, B., Gudziol, H., Steenbeck, J., Freesmeyer, M., Witte, O. W., et al. (2013). Clinical markers of early nigrostriatal neurodegeneration in idiopathic rapid eye movement sleep behavior disorder. Sleep Medicine, 14, 1064-1070.

Sakurai, H., Hanyu, H., Inoue, Y., Kanetaka, H., Nakamura, M., Miyamoto, T., et al. (2014). Longitudinal study of regional cerebral blood flow in elderly patients with idiopathic rapid eye movement sleep behavior disorder. Geriatrics \& Gerontology International, 14, 115-120.

Schenck, C. H., Boeve, B. F., \& Mahowald, M. W. (2013). Delayed emergence of a parkinsonian disorder or dementia in $81 \%$ of older men initially diagnosed with idiopathic rapid eye movement sleep behavior disorder: A 16-year update on a previously reported series. Sleep Medicine, 14, 744-748.

Scherfler, C., Frauscher, B., Schocke, M., Iranzo, A., Gschliesser, V., Seppi, K., et al. (2011). White and gray matter abnormalities in idiopathic rapid eye movement sleep behavior disorder: A diffusion-tensor imaging and voxel-based morphometry study. Annals of Neurology, 69, 400-407.

Shin, H. Y., Joo, E. Y., Kim, S. T., Dhong, H.-J., \& Cho, J. W. (2013). Comparison study of olfactory function and substantia nigra hyperechogenicity in idiopathic REM sleep behavior disorder, Parkinson's disease and normal control. Neurological Sciences, 34, 935-940.

Shirakawa, S. I., Takeuchi, N., Uchimura, N., Ohyama, T., Maeda, H., Abe, T., et al. (2002). Study of image findings in rapid eye movement sleep behavioural disorder. Psychiatry and Clinical Neurosciences, 56, 291-292.

Stiasny-Kolster, K., Doerr, Y., Möller, J. C., Höffken, H., Behr, T. M., Oertel, W. H., et al. (2005). Combination of "idiopathic" REM sleep behaviour disorder and olfactory dysfunction as possible indicator for $\alpha$-synucleinopathy demonstrated by dopamine transporter FP-CIT-SPECT. Brain, 128, 126-137.

Stockner, H., Iranzo, A., Seppi, K., Serradell, M., Gschliesser, V., Sojer, M., et al. (2009). Midbrain hyperechogenicity in idiopathic REM sleep behavior disorder. Movement Disorders, 24, 1906-1909.

Stokholm, M. G., Danielsen, E. H., Hamilton-Dutoit, S. J., \& Borghammer, P. (2016). Pathological $\alpha$-synuclein in gastrointestinal tissues from prodromal Parkinson disease patients. Annals of Neurology, 79, 940-949.

Stokholm, M. G., Iranzo, A., Østergaard, K., Serradell, M., Otto, M., Bacher Svendsen, K., et al. (2018). Extrastriatal monoaminergic dysfunction and enhanced microglial activation in idiopathic rapid eye movement sleep behaviour disorder. Neurobiology of Disease, $115,9-16$.

Stokholm, M. G., Iranzo, A., Østergaard, K., Serradell, M., Otto, M., Svendsen, K. B., et al. (2017). Assessment of neuroinflammation in patients with idiopathic rapid-eyemovement sleep behaviour disorder: A case-control study. The Lancet Neurology, 16, 789-796.

Szewczyk-Krolikowski, K., Menke, R. A. L., Rolinski, M., Duff, E., Salimi-Khorshidi, G., Filippini, N., et al. (2014). Functional connectivity in the basal ganglia network differentiates PD patients from controls. Neurology, 83, 208-214.

Tang, C. C., Poston, K. L., Dhawan, V., \& Eidelberg, D. (2010). Abnormalities in metabolic network activity precede the onset of motor symptoms in Parkinson's disease. Journal of Neuroscience, 30, 1049-1056. 
Unger, M. M., Belke, M., Menzler, K., Heverhagen, J. T., Keil, B., Stiasny-Kolster, K., et al. (2010). Diffusion tensor imaging in idiopathic REM sleep behavior disorder reveals microstructural changes in the brainstem, substantia nigra, olfactory region, and other brain regions. Sleep, 33, 767-773.

Vendette, M., Gagnon, J.-F., Soucy, J.-P., Gosselin, N., Postuma, R. B., Tuineag, M., et al. (2011). Brain perfusion and markers of neurodegeneration in rapid eye movement sleep behavior disorder. Movement Disorders, 26, 1717-1724.

Vendette, M., Montplaisir, J., Gosselin, N., Soucy, J.-P., Postuma, R. B., Dang-Vu, T. T., et al. (2012). Brain perfusion anomalies in rapid eye movement sleep behavior disorder with mild cognitive impairment. Movement Disorders, 27, 1255-1261.

Vilas, D., Iranzo, A., Pont-Sunyer, C., Serradell, M., Gaig, C., Santamaria, J., et al. (2015). Brainstem raphe and substantia nigra echogenicity in idiopathic REM sleep behavior disorder with comorbid depression. Journal of Neurology, 262, 1665-1672.

Wing, Y. K., Lam, S. P., Zhang, J., Leung, E., Ho, C. L., Chen, S., et al. (2015). Reduced striatal dopamine transmission in REM sleep behavior disorder comorbid with depression. Neurology, 84, 516-522.

Wu, P., Yu, H., Peng, S., Dauvilliers, Y., Wang, J., Ge, J., et al. (2014). Consistent abnormalities in metabolic network activity in idiopathic rapid eye movement sleep behaviour disorder. Brain, 137, 3122-3128.

Zhang, L., Xu, Y., Zhuang, J., Peng, H., Wu, H., Zhao, Z., et al. (2016). Metabolic abnormality of pontine tegmentum in patients with REM sleep behavior disorder analyzed using magnetic resonance spectroscopy. Clinical Neurology and Neurosurgery, 148, 137-141.

Zhu, R. L., Xie, C. J., Hu, P. P., \& Wang, K. (2017). Clinical variations in Parkinson's disease patients with or without REM sleep behaviour disorder: A meta-analysis. Scientific Reports, 7, 40779. http://doi:10.1038/srep40779.

Zoetmulder, M., Nikolic, M., Biernat, H., Korbo, L., Friberg, L., \& Jennum, P. (2016). Increased motor activity during REM sleep is linked with dopamine function in idiopathic REM sleep behavior disorder and Parkinson disease. Journal of Clinical Sleep Medicine, 12, 895-903. 